\title{
Relationship between Balance of Upper and Lower Lip-Closing Forces during Pursing-like Lip-Closing and Lateral Craniofacial Morphology
}

\author{
Madoka Murakami ${ }^{1}$, Masanori Kanazawa ${ }^{2}$, Rurika Mizuno ${ }^{3}$, Kazuhiro Yamada ${ }^{4}$, Yuji \\ Masuda ${ }^{5}$ and Tadafumi Adachi ${ }^{6}$ \\ ${ }^{1,2,3,4}$ Department of Orthodontics, Matsumoto Dental University, Shiojiri, Nagano, Japan \\ 5,6 Department of Oral and Maxillofacial Biology, Graduate School of Oral Medicine, Matsumoto Dental \\ University, Shiojiri, Nagano, Japan
}

Correspondence should be addressed to: Yuji Masuda; masuday@po.mdu.ac.jp

Received date: 23 April 2014; Accepted date: 20 May 2014; Published date: 11 August 2014

Academic Editor: Jiuxiang Lin

Copyright (C) 2014. Magda Mohasseb and Gihane Ibrahim Khalil. Distributed under Creative Commons CC-BY 3.0

\begin{abstract}
The aim of this study was to clarify the relationship between the balance of upper- and lower-lip-closing forces (LCF) during pursing-like lip-closing and lateral craniofacial morphology. Sixty-two females with malocclusion were subjected to lateral cephalography and divided into 3 groups (skeletal Classes I, II and III) by the value of ANB. The LCF was measured using a multidirectional LCF measurement system, which could assess eight directional LCF during pursing-like lip-closing. Statistical analysis by the comparison of values among the groups was performed by the Kruskal-Wallis test, and correlation coefficients were tested by Pearson's product-moment correlation. Significance was set at $\mathrm{P}<0.05$.

Total upper LCF, which was the sum of upper, right and left upper directional LCF, in Class II was significantly greater than that in Class I. The balance index of upper and lower LCF in group II was significantly higher than that in group III. There were weak positive correlations between upper LCF and FMA, and the balance index of upper and lower LCF and ANB or convexity, whereas there was a negative correlation between FMIA and the balance index of upper and lower LCF.

These findings support the concept of dental compensation for anterior-posterior malposition of the maxillary and mandibular regions by inclination of the incisors.
\end{abstract}

Keywords: lip-closing force, multidirectional measurement system, lateral craniofacial morphology

\section{Introduction}

It is widely recognized that the balance of forces on the teeth from the lips and tongue is one of the most important factors determining the position of the dental arch (Ahlgren JG et al, 1973, Lowe AA et al, 1984, Jung MH et al, 2003). In other words, lip force can affect craniofacial

Cite this Article as: Madoka Murakami, Masanori Kanazawa, Rurika Mizuno, Kazuhiro Yamada, Yuji Masuda and Tadafumi Adachi(2014), "Relationship between Balance of Upper and Lower Lip-Closing Forces during Pursing-like Lip-Closing and Lateral Craniofacial Morphology", Journal of Research and Practice in Dentistry, Vol 2014 (2014), Article ID 171681, DOI: 10.5171/2014.171681 
morphology. This was proposed in the 1950s as the buccinator mechanism in orthodontics (Brodie AG., 1953, Moyers RE., 1958). The effects of lip-closing force (LCF) on dentition have been applied for orthodontic treatments such as myofunctional therapy.

Many studies have attempted to clarify the relationship between the LCF of subjects and their malocclusion using various systems for measuring LCF. For example, by using a dynamometer, LCF was measured as vertical lip force for upper and lower lips, the same as with the Myometer 160 (Ingervall B et al, 1981, Posen AL., 1976, Lambrechts $\mathrm{H}$ et al., 2010). Another measurement system involved the lip pressure on the incisors (Thuer $U$ et al., 1986). Jung et al, (2003 and 2010) attempted to measure the upper- and lower-lip forces separately using a Y-meter. However, these studies did not obtain consistent findings on the relationship between LCF and malocclusion because of the different measurement methods and developmental stages of subjects.

The orbicularis oris muscle (OOM), which plays a dominant role in producing $\mathrm{LCF}$, is an orbicular muscle surrounding the oral slit and does not function in a simple manner. It is innervated by the facial nerve, mainly unilaterally. In addition, it is comprised of two different components: one is the buccal branch in the upper labial part and the other is the mandibular branch in the lower labial part. In addition, the upper and lower parts of the OOM have different functional properties in force development, stability and fatigability (Amerman JD., 1993, Barlow SM et al., 1996, Gentil M et al,, 1998).

These characteristics of the 00M enable various patterns of LCF to be performed, corresponding to particular functions. It is thought that, when we maintain static force control of the lips, the multidirectional outputs of LCF can be controlled by precise cooperation between numerous contacts surrounding the lips. From this perspective, the balance of different directional LCF may affect the morphology.

We have developed a multidirectional LCF measurement system that allows us to measure the LCF in eight different directions during pursing-like lip-closing (Nakatsuka $\mathrm{K}$ et al, 2011a), and we reported the properties of multidirectional LCF for healthy young adults, as well as postoperative patients with cleft lip and/or palate (Nakatsuka K et al, 2011abc, Murakami $\mathrm{M}$ et al, 2012). We demonstrated the ability to analyze the directional balance of LCF using multidirectional LCF.

Alteration of the dental axis was shown to compensate for the occlusal function when the anterior-posterior position between maxillary and mandibular regions is incorrect skeletally. This phenomenon called "dental compensation" has been frequently observed in clinical dentistry. However, the mechanism that causes it has remained unclear.

We hypothesized that an imbalance in upper and lower lip forces affecting teeth position and/or inclination does not result in malocclusion, but rather in dental compensation, in young adults with skeletal malocclusion at a late stage of development. The aim of this study was to test this hypothesis by measuring multidirectional LCF during pursing-like lip-closing and lateral craniofacial morphology.

\section{Materials and Methods}

\section{Subjects}

This research was approved by the Ethics Committee on Clinical Research of Matsumoto Dental University (Approval Number: 0057). Sixty-two females (median $=23.0$ years, range $=$ 17-34 years) were recruited from among patients with malocclusion who were referred to the Department of Orthodontics of Matsumoto Dental University Hospital. None had a history of lip injury or neurological disease. This study excluded those with a remarkable lateral shift of the mandible (magnitude of shift of the menton larger than $4.0 \mathrm{~mm}$ on a frontal cephalogram), those with previous experience of orthodontic treatment and those with missing teeth, including the congenital lack of teeth (apart from third molars). All subjects received verbal and written information about the study and gave verbal consent to participate.

\section{Classification of subjects}

In all subjects, standardized lateral cephalometric radiographs were taken with the lips closed and centric occlusion. From their lateral cephalograms, the subjects were divided into 3 groups (skeletal Classes I, II and III) using the 
value of ANB as follows:

(1) skeletal Class I: ANB $2^{\circ}-4^{\circ}$;

(2) skeletal Class II: $\mathrm{ANB}>4^{\circ}$;

(3) skeletal Class III: $\mathrm{ANB}<2^{\circ}$.

\section{Analysis of lateral cephalogram}

Skeletal, dental and soft-tissue cephalometric landmarks were determined using software (Win Ceph $^{\circledR}$ version 9.0, Rise Co., Miyagi, Japan).
In order to determine the characteristics of the facial skeleton and the inclination of anterior teeth, we measured 11 angles and 4 lengths from the reference points and lines of the lateral cephalogram (Fig. 1).

In order to determine the measurement error, cephalograms of ten subjects chosen at random were traced twice at an interval of two weeks and the error was calculated using Dahlberg's formula (Dahlberg G., 1940).

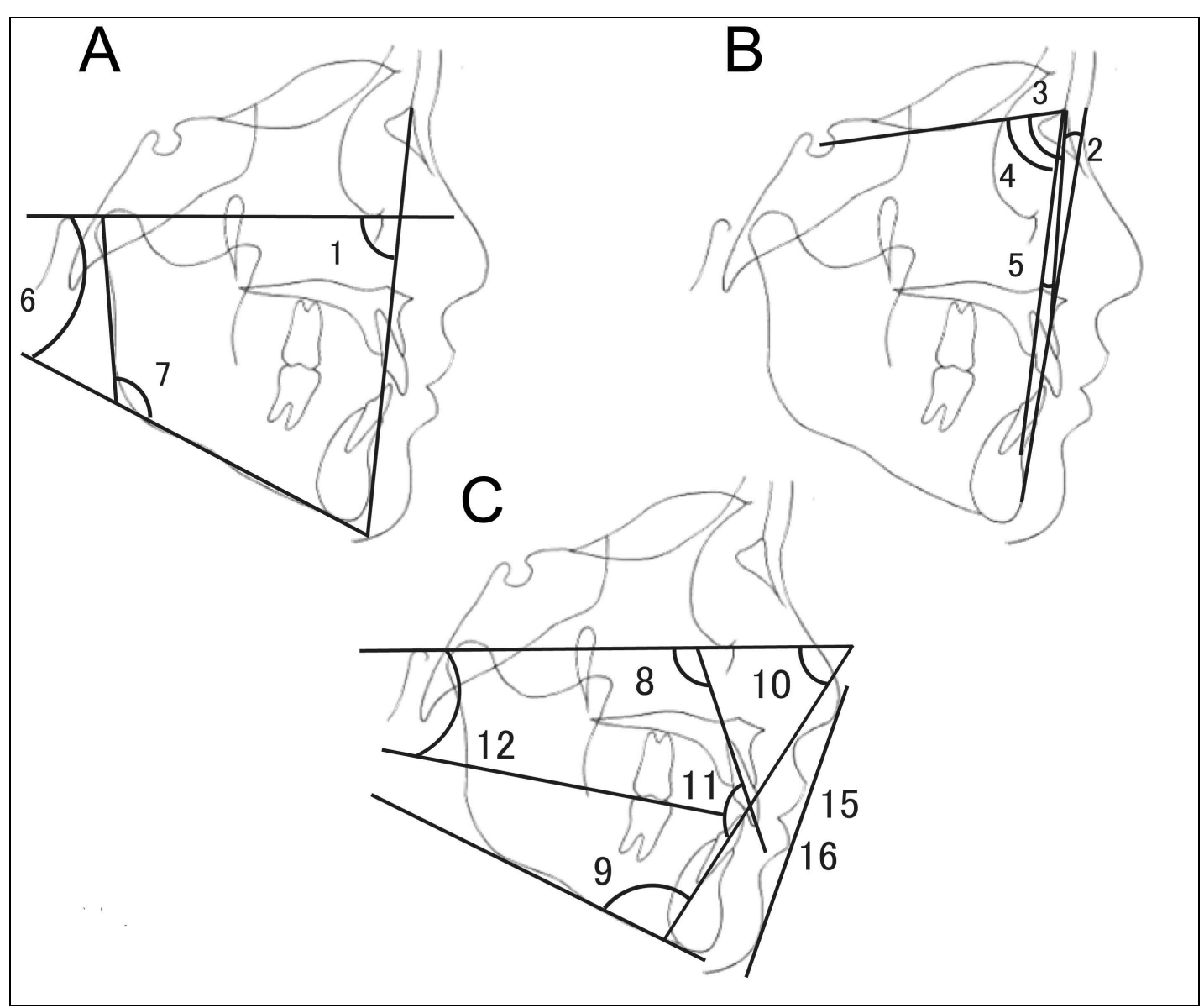

Fig. 1: Analysis of lateral cephalogram

A, B: Skeletal cephalometric landmarks: 1, Faci

al angle; 2, Convexity; 3, SNA, 4, SNB; 5, ANB; 6, FMA; and 7, Gonial angle.

C: Dental cephalometric landmarks: 8, U1-FH plane angle; 9, IMPA; 10, FMIA; 11, Interincisal angle; 12, Occlusal plane angle; 13, Overjet; and 14, Overbit

Soft-tissue cephalometric landmarks: 15, E-line (UL); and 16, E-line (LL) 


\section{Multidirectional lip-closing force}

\section{Design of instrumentation}

A multidirectional LCF measurement system (Patent Numbers: JP-4487132, JP-B-4554630) was used to measure LCF in this study. A detailed description of this apparatus has been provided elsewhere (Nakatsuka $\mathrm{K}$ et al, 2011a). .

Data acquisition and analysis were performed using customized waveform management software for the multidirectional lip-closing force measurement system. Using this software, the lip-force signals digitized at $100 \mathrm{~Hz}$ and obtained from eight directions could be simultaneously displayed on a computer screen and saved on a personal computer. The software also enabled the quantification of LCF in a single direction (directional LCF: DLCF).

\section{Maximal voluntary LCF measuring procedure}

Before the recording, the subjects were given a brief explanation of the apparatus and the experimental lip tasks by the investigators. They were seated on a chair with support for their knees in a comfortable position. Each subject's head was fixed so that the Camper plane line was parallel to the horizontally adjusted measurement probe (Fig. 2). After a few practice runs, the recording session was started. For each trial, the subjects pursed their lips as hard as possible with the probe between their upper and lower lips, but without tooth contact, for 5-6 s. Each set consisted of three trials with intervals of at least 5 seconds.

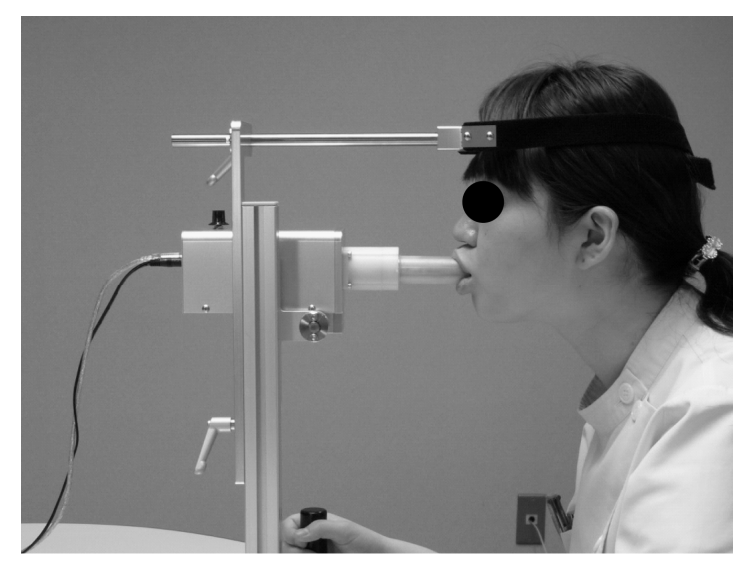

Figure 2: Multidirectional LCF measurement system (Patent Numbers: JP-4554630)

Each subject's head was fixed so that the Camper plane line was parallel to the horizontally adjusted measurement probe.

\section{Data analysis}

The DLCF signals in all eight directions became stable $1 \mathrm{~s}$ after the onset of the signals during the pursing-like lip-closing. The DLCF in each direction was assessed using the impulse value [force (N) multiplied by time (s)]. The mean impulse (impulse per second) was calculated between 1 and 4 seconds after the onset of the LCF (Nakatsuka $\mathrm{K}$ et al, 2011c). The DLCF values obtained from six recordings ( 3 trials $\mathrm{x} 2$ sets) were averaged for each session in each subject. Total lip closing force (TLCF) was calculated by summing the DLCF recorded in the eight directions.

In addition, in this study, asymmetric comparison between upper lip and lower LCF was performed. In other words, as the balance of the upper and lower LCF, the total upper LCF (TULCF) was calculated as the sum of the DLCF from the upper, right upper and left upper directions, and the total lower LCF (TLLCF) was calculated as the sum of the DLCF from the lower, right lower and left lower directions. The balance index of upper and lower LCF was the 
value of the differences between the total upper and lower LCF divided by the sum of them. We examined the correlation coefficients between TLCF, TULCF, TLLCF and the balance index of upper and lower LCF and lateral craniofacial morphology.

\section{Statistical analysis}

Statistical analysis was performed using the Kruskal-Wallis test for the comparison of TLCF among malocclusion types of skeletal Classes I, II and III, and Mann-Whitnney test with Bonferroni correction was used for post hoc test to test the significance of differences between two samples. Pearson's product-moment correlation was used to identify significant associations of TLCF or balance of the upper- and lower-lip-closing forces from the lateral cephalograms for patients with malocclusion. Statistical analysis was performed with SPSS for Windows 14.0 J (SPSS Co. Ltd., Tokyo, Japan). The data are presented as the mean \pm s.d. P-values $<0.05$ were considered significant.

\section{Results}

\section{Analysis of the lateral cephalograms}

In this study, the measurement error determined by Dahlberg's formula (Table 1) was very small (Houston W J B., 1983); the results for angular analysis were 0.10-0.31 degrees, and those for linear analysis were $0.10-0.20 \mathrm{~mm}$. These findings indicate that the analysis of the lateral cephalograms in this study was reliable because previous studies showed that methodological errors of cephalometric variables were less than $1.0 \mathrm{~mm}$ for linear measurements and $1.5^{\circ}$ for angular measurements.

Table 1: Dahlberg's formula All cephalometric variables were less than $1.0 \mathrm{~mm}$ for linear measurements, and $1.5^{\circ}$ for angular measurements.

\begin{tabular}{|c|c|}
\hline & $\begin{array}{l}\text { Dahlberg's } \\
\text { formula }\end{array}$ \\
\hline Facial angle & 0.10 \\
\hline Convexity & 0.15 \\
\hline SDA & 0.14 \\
\hline SDAB & 0.15 \\
\hline$A N B$ & 0.05 \\
\hline FNA & 0.15 \\
\hline Gonial amgle & 0.15 \\
\hline U-1 to FII plane & 0.28 \\
\hline INPA & 0.32 \\
\hline FNIA & 0.29 \\
\hline Interincisal & 0.27 \\
\hline Occlusal plame & 0.20 \\
\hline Overjet & 0.15 \\
\hline Overbite & 0.14 \\
\hline E-line (UI) & 0.10 \\
\hline E-line (II) & 0.11 \\
\hline
\end{tabular}




\section{Classification of subjects}

From the results of our skeletal Classification by ANB, subjects were classified into three groups as follows: 20 females (median $=22.3$ years) with skeletal Class I, 22 females (median $=22.3$ years) with skeletal Class II and 20 females ( median $=22.0$ years) with skeletal Class III.

\section{Comparison of LCF among three Classes}

Fig. 3 shows the LCF and the calculated variables of LCF in the three Classes. TLCF was $3.72 \pm 1.31$ Ns in skeletal Class I, 4.96 \pm 2.71 Ns in Class II and $5.44 \pm 3.03$ Ns in Class III (Fig. 3A). It did not show a significant difference among these three groups. Significant inter-group differences were observed in upper DLCF, TULCF and the balance index of upper and lower LCF. The TULCF in Class II was significantly greater than that in Class I (Fig. 3B). The balance index of upper and lower LCF in Class II was significantly higher than that in Class III (Fig. 3C)

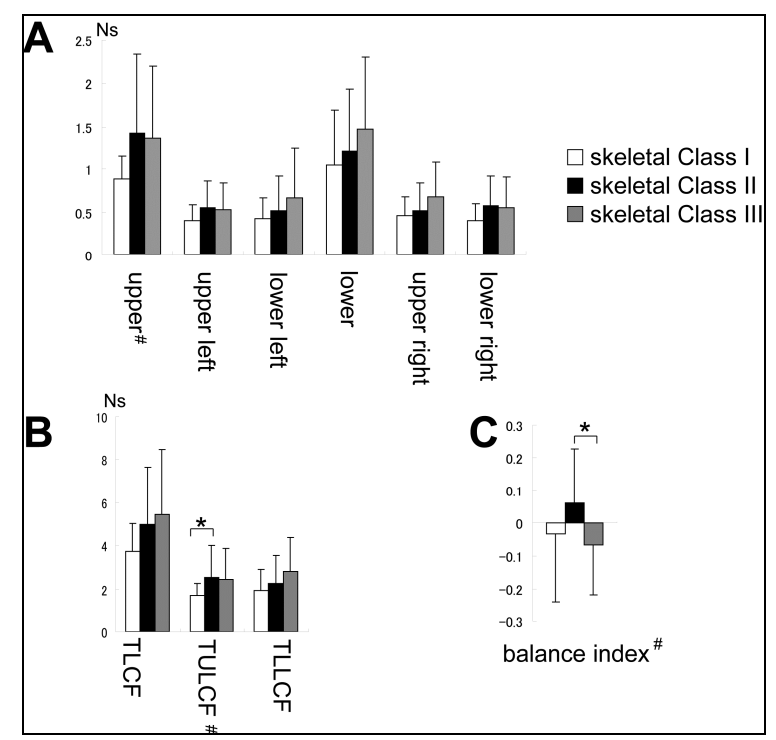

Fig. 3: LCF and calculated variables of LCF in the three Classes

A: Eight DLCFs in the three Classes. There was a significant difference among the three Classes in upper DLCF.

B: TLCF, TULCF and TLLCF in the three Classes. The total upper LCF in Class II was significantly larger than that in Class I.

C: Balance index in the three Classes. The upper and lower LCF in Class II were significantly larger than those in Class III. \#: p $<0.05$ (Kruskal Wallis test), *: $\mathbf{p}<0.05$ (post hoc test)

Correlation coefficients between LCF and measurements from lateral cephalograms

For analyzing the correlation coefficients, we used the measurements that indicate the skeletal relationship between maxillary and mandibular regions, as well as the incisor axis. The variables of LCF that showed significant inter-group 
differences were used. As shown in Table 2, there were weak positive correlations between the upper LCF and FMA ( $\mathrm{r}=0.257, \mathrm{P}<0.05)$, and the balance index of upper and lower LCF and
ANB ( $r=0.262, \mathrm{P}<0.05)$ or convexity $(r=0.307$, $\mathrm{P}<0.05)$, whereas there was a negative correlation between FMIA and the balance index of upper and lower LCF ( $\mathrm{r}=-0.262, \mathrm{P}<0.05)$.

Table 2: Correlation coefficients between LCF and measurements from lateral cephalograms

There were weak positive correlations between upper DLCF and FMA, and the balance index of upper and lower LCF and ANB or convexity, whereas there was a negative correlation between FMIA and the balance index of upper and lower LCF.

\begin{tabular}{|c|c|c|c|c|c|}
\hline & \multicolumn{5}{|c|}{ Correlation coefficient } \\
\hline & & Upper & TULCF & Balance In & dex \\
\hline Facial angle & - & 0.034 & - 0.008 & - 0.204 & \\
\hline Convexity & & 0.131 & 0.143 & 0.307 & $*$ \\
\hline SNA & & 0.088 & 0.093 & 0.072 & \\
\hline SNB & - & 0.002 & - 0.012 & - 0.147 & \\
\hline ANB & & 0.104 & 0.122 & 0.262 & * \\
\hline FMA & & $0.257 *$ & 0.237 & 0.234 & \\
\hline Gonial angle & & 0.126 & 0.130 & 0.092 & \\
\hline $\mathrm{U}-1$ to $\mathrm{FH}$ plane & - & 0.183 & - 0.199 & - $\quad 0.069$ & \\
\hline IMPA & & 0.202 & - 0.206 & 0.090 & \\
\hline FMПA & & 0.019 & 0.036 & - 0.262 & $*$ \\
\hline Interincisal & & 0.156 & 0.182 & - 0.132 & \\
\hline Occlusal plane & & 0.177 & 0.141 & 0.168 & \\
\hline Overjet & - & 0.112 & - 0.118 & - 0.073 & \\
\hline Overbite & - & 0.020 & - 0.038 & 0.012 & \\
\hline E-line (UL) & - & 0.165 & - 0.178 & 0.120 & \\
\hline E-line (LL) & - & 0.141 & - 0.146 & 0.131 & \\
\hline
\end{tabular}

\section{Discussion}

\section{TLCF}

Many previous studies showed the relationship between Angle classification and lip force. Posen (1976) measured high lip strength with maximum voluntary contraction using a pommeter (perioral muscle meter). Lip pressure on the incisors of subjects with Angle Class II div. 2 was high, and that on the incisors of subjects with Angle Class II div. 1 was lower than those with Angle Class I. Lambrechts et al, (2010) also obtained the same results using Myometer 160, which measured the LCF generated between upper and lower lips at the 
midline during lip-closing.

Those studies assessed lip force generated by the whole lip. Therefore, we accessed total LCF, namely, the sum of 8 directional LCF during pursing-like lip-closing, for comparison with these previous results. TLCF did not differ among skeletal Classes I, II and III in this study. This may have depended on the different classifications by skeletal pattern. U1 to FH of subjects with skeletal Class II in this study ranged from $81.0^{\circ}$ to $131.7^{\circ}$. This means that skeletal Class II in our study might include both Angle Class II div. 1 and 2.

The previous results showing that the lip force of subjects with Angle Class II div. 1 was weaker and that of subjects with Angle Class II div. 2 was greater may be related to the angulation of upper incisors in previous studies, but not the skeletal pattern.

\section{Upper- and lower-lip forces}

The results obtained using a Y-meter showed the upper-and lower-lip forces separately. Therefore, by our methods, TULCF and TLLCF, namely, the sums of the 3 upper and 3 lower DLCFs, respectively, were used for upper- and lower-lip forces for comparison with the results obtained using a Y-meter. The TULCF in skeletal Class II was greater than that in skeletal Class I. This result is similar to previous results of lip pressure on the upper incisors in children (Thuer $U$ et al., 1986). The lip pressure on the upper incisors was higher in Angle Class II div. 1 than in Angle Class I. These findings suggest that the pressure from the lips on the teeth may cause lingual angulation of the upper incisors in children. This incisor angulation may be involved in dental compensation in adults. The same study showed that the lip pressure on the upper incisors was lowest in Angle Class II div. 2 in children. This seemed to show that our subjects in skeletal Class II tended not to have incisors with lingual angulation. Indeed, the mean of the U1-FH plane angle was $107.8^{\circ}$ in our skeletal Class II subjects, which is within the normal range.

The upper-lip force was greater than the lower-lip force in all subjects included in Angle Class I, and Angle Class II div. 1 and 2.10 Furthermore, in Angle Class I and II subjects, the values of the maximum lip-closing forces were related to the variables of upper-incisor angulation. Those lip forces might be a cause of dental compensation. In our measurement methods, upper-lip force was similar to lower-lip force owing to the difference of movement during measurement. Specifically, we measured the LCF during pursing-like lip-closing, although with the Y-meter, the lip force was measured during movement like lip-sealing with maximum effort.

\section{Balance of upper and lower LCF}

For the purpose of this study, we classified the subjects as having a skeletal pattern of dental compensation for those with skeletal malocclusion. In this study, upper- and lower-lip forces were estimated using the balance of upper and lower LCF.

This study revealed that the balance index of upper and lower LCF showed positive correlations with ANB and convexity, and a negative correlation with FMIA. In short, in subjects of skeletal Class II, in which mandibular bone is positioned posterior to maxillary bone, upper-lip force is greater than lower-lip force, and this balance of lip force is considered to cause labial inclination of the lower incisors. In subjects of skeletal Class III, in which mandibular bone is positioned anterior to maxillary bone, lower-lip force is greater than upper-lip force, and this balance of lip force is considered to cause lingual inclination of the lower incisors. These findings support the concept of dental compensation, that is, compensation for the anterior-posterior malposition of the maxillary and mandibular regions by inclination of the incisors. The other result of the total upper LCF in group II being significantly greater than that in the other groups also supports the concept of dental compensation.

In order to prevent relapse, it is necessary to determine the muscle forces of soft tissues in the preoperative period. This study suggests that muscle activity can cause dental compensation and may provide useful information for orthodontic treatment, including the prevention of relapse.

\section{Conclusions}

There were weak positive correlations between upper LCF and FMA, and the balance index of upper and lower LCF and ANB or convexity. In addition, there was a negative correlation 
between FMIA and the balance index of upper and lower LCF. These findings support the concept of dental compensation, that is, compensation of the anterior-posterior malposition of the maxillary and mandibular regions by inclination of the incisors.

\section{References}

1. Ahlgren, J. G., Ingervall, B. F. and Thilander, B. L. (1973) "Muscle activity in normal and postnormal occlusion," American Journal of Orthodontics, 64 (5) 445-456.

2. Amerman, J. D. (1993) "A maximum-force-dependent protocol for assessing labial force control," Journal of Speech and Hearing Resarch, 36 (3) 460-465.

3. Barlow, S. M. and Bradford, P. T. (1996) "Comparison of perioral reflex modulation in the upper and lower lip, " Journal of Speech and Hearing Research, 39 (1) 55-75.

4. Brodie, A. G. (1953) "Muscular Factors in the Diagnosis and Treatment of Malocclusions," Angle Orthod, 23 (4) 71-77.

5. Dahlberg, G. (1940) Satistical methods for medical and biological students, Interscience Publications, New York, USA.

6. Gentil, M. and Tournier, C. L. (1998) "Differences in fine control of forces generated by the tongue, lips and fingers in humans," Archives of Oral Biology. 43 (7) 517-523.

7. Houston, W. J. B. (1983) "The analysis of errors in orthodontic measurements,"

American Journal of Orthodontics. 83 (5) 382-390

8. Ingervall, B. and Janson, T. (1981) "The value of clinical lip strength measurements," American Journal of Orthodontics. 80 (5) 496-507.

9. Jung, M. H., Yang, W. S. and Nahm, D. S. (2003) "Effects of upper lip closing force on craniofacial structures," American Journal of Orthodontics and Dentofacial Orthopedics. 123 (1) 58-63.

10. Jung, M.H., Yang, W.S. and Nahm, D.S. (2010) "Maximum Closing Force of Mentolabial
Muscles and Type of Malocclusion," Angle Orthod. 80 (1) 72-79.

11. Lambrechts, H., Baets, E. D., Fiews, S. and Willems, G. (2010) "Lip and tongue pressure in orthodontic patients," European Journal of Orthodontics. 32 (1) 466-471.

12. Lowe, A. A. and Takada, K. (1984) "Associations between anterior temporal, masseter, and orbicularis oris muscle activity and craniofacial morphology in children," American Journal of Orthodontics. 86 (4) 319-330.

13. Moyers, R. E. (1958) Handbook of Orthodontics, The Year Book Publishers, Chicago, USA.

14. Murakami, M., Adachi, T., Nakatsuka, K., Kato, T., Oishi, M. and Masuda, Y. (2012) "Gender differences in maximum voluntary lip-closing force during lip pursing in healthy young adults, " Journal of Oral Rehabilitation. 39 (6) 399-404.

15. Nakatsuka, K., Adachi, T., Kato, T., Oishi, M., Murakami, M., Okada, Y. and Masuda, Y. (2011a) "Reliability of novel multidirectional lip-closing forcemeasurement system," Journal of Oral Rehabilitation. 38 (1) 18-26.

16. Nakatsuka, K., Adachi, T., Kato, T., Oishi, M. and Masuda, Y. (2011b) "Regulatory relationship between tactile sensation at the vermilion of the lips and lip-closing force," Journal of Oral Rehabilitation. 38 (8) 579-587.

17. Nakatsuka, K., Adachi, T., Kato, T., Murakami, M., Yamada, K. and Masuda, Y. (2011c) "Asymmetric lip-closing forces in children with repaired unilateral cleft lip and/or palate," Journal of Oral Rehabilitation. 38 (12) 921-928.

18. Posen, A. L. (1976) "The application of quantitative perioral assessment to orthodontic case analysis and treatment planning," Angle Orthod. 46 (2) 118-143.

19. Thuer, U. and Ingervall, B. (1986) "Pressure from the lips on the teeth and malocclusion," American Journal of Orthodontics and Dentofacial Orthopedics. 90 (3) 234-2. 